\title{
Management of Inflamed Conjunctival Nevus with Topical Antiallergic Medications: Case Series
}

This article was published in the following Dove Press journal:

Clinical Ophthalmology

\section{Kumiko Kato (iD ${ }^{\prime}$ \\ Maki Takeuchi' \\ Yuka Yonekawa' \\ Yuzen Kashima' \\ Koji Hirano $\mathbb{D}^{2}$ \\ Mineo Kondo'}

'Department of Ophthalmology, Mie University Graduate School of Medicine, Tsu, Japan; ${ }^{2}$ Department of

Ophthalmology, Fujita Health University Bantane Hospital, Nagoya, Japan
Correspondence: Kumiko Kato Department of Ophthalmology, Mie University Graduate School of Medicine, 2-I74 Edobashi, Tsu, Mie, 5 14-8507, Japan Tel +8I-59-23I-5027

Fax +8I-59-23I-3036

Email k-kato@clin.medic.mie-u.ac.jp
Purpose: To report our findings in three cases of an inflamed conjunctival nevus whose size and degree of pigmentation were reduced by topical antiallergic and immunosuppressive ophthalmic solutions.

Methods: Observational case series. Three patients with inflamed conjunctival nevus were examined by slit-lamp biomicroscopy, and the findings before and after the treatments were compared.

Results: All three patients had a slightly pigmented and edematous conjunctival tumor at the corneal limbal area. The tumors were hyperemic, and papillae and follicles were present on the superior conjunctiva in all patients. All of the patients had an allergic predisposition. The antiallergic treatment not only resolved the hyperemia and edema of the palpebral conjunctiva, but also reduced the tumor size. In one case, the topical antiallergic agent alone led to a reduction of the tumor size. A combination of topical antiallergic agent and topical immunosuppressant was effective in reducing the tumor size and degree of pigmentation in the other two patients.

Conclusion: The hyperemia and pigmentation in eyes with inflamed conjunctival nevus can be resolved by topical antiallergic agent and topical immunosuppressant without resection of the tumor.

Keywords: inflamed juvenile conjunctival nevus, epinastine, tacrolimus

\section{Introduction}

Inflamed juvenile conjunctival nevi (IJCN) are relatively common conjunctival tumors that develop at puberty and young adulthood. IJCN are benign, usually located near the limbus and are amelanotic. ${ }^{1}$ They are often vascularized by large feeder vessels. Zamir et al reported that all patients with IJCN had histories and physical findings of systemic allergy such as atopic dermatitis, asthma, bilateral papillary conjunctivitis, and vernal conjunctivitis. ${ }^{2}$ IJCN are benign conjunctival tumors, and they are generally managed by observation alone. However, excision is recommended if the tumor has any signs of malignancy, or if they cause tear film instability that results in corneal thinning, or if they interfere with contact lens wear. Alternatively, resection should be considered if the patients and/or their families are concerned about the nature of the tumor and the cosmetic appearance. However, tumor resection under local anesthesia is often difficult in pediatric patients, so we believe that observations with close follow-up may be desirable until the patient is old enough to tolerate the local anesthesia and surgery.

A search of PubMed for treatments for IJCN did not extract any publications on successful treatments for IJCN other than resection. ${ }^{3}$ We have examined three cases 
of IJCN in which topical application of an antiallergic agent, eg, antihistamine, and an immunosuppressive drug, eg, tacrolimus, led to a marked reduction in the size and degree of pigmentation of the IJCN.

\section{Methods}

This was an observational case series that included patients who were diagnosed with IJCN at the Mie University Hospital. All patients or legal guardians provided informed consent for the case details, and also for any accompanying images to be published according to the tenets of the Declaration of Helsinki. Ethical approval was waived by the Institutional Ethics Committee of Mie University Hospital, because of the retrospective nature of the case series that was limited to collecting clinical data with no personal identifiers of the patient being recorded.

The diagnosis of IJCN was based on the following clinical findings: conjunctival tumor located near the limbus, presence of allergic conjunctivitis determined by slit-lamp biomicroscopy, presence of feeder vessels, and conjunctival tumor size fluctuating with the severity of allergic conjunctivitis. In this case series, we used the term of "feeder vessel" to refer to blood vessels that were larger than the surrounding blood vessels.
Patients were treated with antiallergic agents alone or in combination with either tacrolimus or low-concentration steroids. We defined the end point of treatment in IJCN as a reduction in the tumor size.

\section{Case Presentations}

\section{Case I}

An 8-year-old boy came to our hospital complaining of a rapid growth of a tumor on his left eye. He stated that he had allergic rhinitis and allergic conjunctivitis and was using topical $0.1 \%$ olopatadine (Novartis Pharma K.K. Tokyo, Japan) 4 times/day for the allergic conjunctivitis. Slit-lamp biomicroscopy showed an elevated conjunctival lesion with edema, hyperemia, and petechial pigmented lesion (Figure 1A). A feeder vessel to the tumor was observed (Figure 1A). There was papillary and follicular conjunctivitis on the superior palpebral conjunctiva. We switched his medications from topical $0.1 \%$ olopatadine to topical $0.05 \%$ epinastine (Santen Pharmaceutical Co., Ltd, Osaka, Japan) 4 times/day and added topical $0.1 \%$ tacrolimus (Senju Pharmaceutical Co., Ltd, Osaka, Japan), an immunosuppressant 2 times/day, to treat the allergic conjunctivitis. A month later, the elevation and edema of the conjunctival lesion had diminished (Figure 1B). We continued the treatment with topical

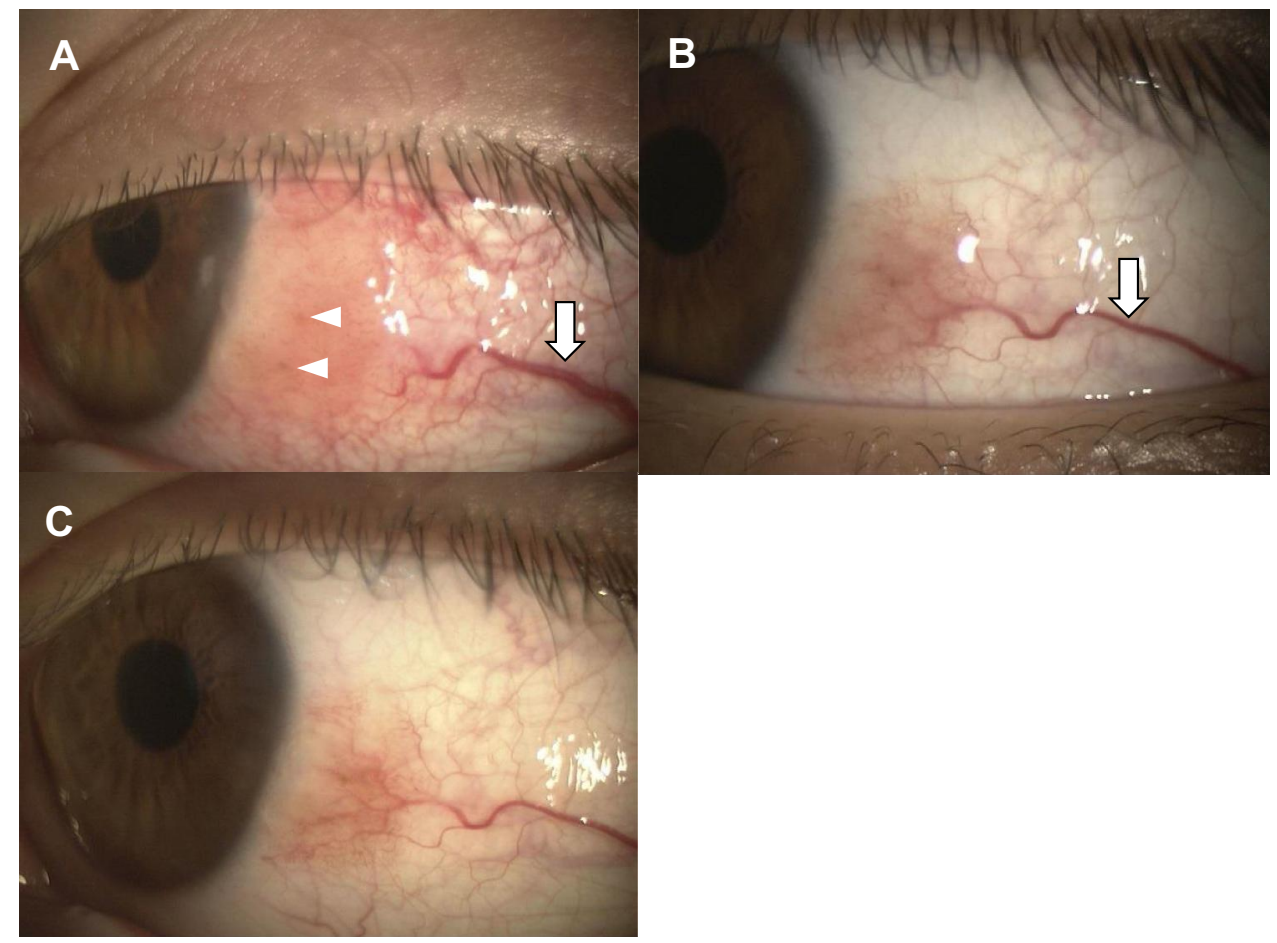

Figure I Slit-lamp biomicroscopic findings in Case I. (A) Conjunctival tumor can be seen at the corneal limbus with edema and feeder vessel (arrow). A petechial pigmented lesion can be seen (arrowheads). (B) Photograph of eye one month after treatment with topical epinastine and tacrolimus, an immunosuppressive drug. The tumor size and edema are markedly reduced, and the feeder vessel has regressed (arrow). (C) One year after the treatment. The tumor is not detectable. 
epinastine and tacrolimus. At 1 year, the size of the tumor was further reduced, and the degree of pigment was decreased (Figure 1C). In the past, the eye drops had been discontinued, but due to significant exacerbation of the tumor, the eye drops were resumed and have been continued to the present. We follow him for seven years, and there has been no sign of malignancy.

\section{Case 2}

A 14-year-old boy was referred to our hospital with a suspicion of a malignant tumor on the conjunctiva of his left eye. He had been diagnosed with allergic conjunctivitis but was not treated because he had no symptoms. Slit-lamp biomicroscopy showed an elevated pigmented lesion and edema in the nasal limbal conjunctiva (Figure 2A). Feeder vessels to the tumor were observed (Figure 2A). In addition, the superior palpebral conjunctiva was hyperemic with papillary and follicular changes. We began his treatment with topical $0.05 \%$ epinastine 4 times/day and one month later, the edema diminished (Figure 2B). We have followed him for one year while continuing the $0.05 \%$ epinastine 4 times/day, and there have been no signs of malignancy at the final visit.

\section{Case 3}

A 34-year-old man was diagnosed with atopic dermatitis with hyperemia of the right eye when he was a child. Because of a sudden aggravation of the hyperemia, he visited another eye clinic and was treated with topical $0.1 \%$ betamethasone (Shionogi \& Co., Ltd, Osaka, Japan) 4 times/day. He was referred to our hospital because the betamethasone was ineffective, and he was suspected of having a malignant melanoma. Slit-lamp biomicroscopy showed severe hyperemia and petechial pigmented lesion in the limbal area of the right eye (Figure 3A). The superior palpebral conjunctiva was hyperemic with follicular changes indicating complications of allergic conjunctivitis. His IOP was elevated due to the topical betamethasone, and we switched him to topical $0.05 \%$ epinastine 4 times/day and $0.1 \%$ fluorometholone (Santen Pharmaceutical Co., Ltd, Osaka, Japan), a corticosteroid 4 times/day. One month later, we found an increase in the conjunctival lesion and exacerbation of the pigmentation and hyperemia (Figure 3B) Feeder vessels to the lesion were observed (Figure 3B). We switched the treatment to topical $0.05 \%$ epinastine and topical $0.1 \%$ tacrolimus, and 1 month later, the conjunctival lesion was markedly reduced, and the pigmentation was barely visible (Figure 3C).

\section{Discussion}

We examined three cases of conjunctival tumor in which we were able to observe a decrease in the tumor size and/ or the pigmentation. We did not perform histopathological examinations because of the successful treatment with topical medication and the patients did not wish to have excisional biopsy. However, we suspected they might be IJCN based on the age of onset, the location of the tumor, and presence of feeder vessels.

Conjunctival tumors can have either benign or malignant features. Shields et al studied 806 cases of conjunctival tumors in young adults ( $<21$ years) retrospectively and reported that $97 \%$ were benign and $3 \%$ were malignant. ${ }^{4}$ The five most common benign conjunctival tumor were nevus $(61 \%)$, benign reactive lymphoid hyperplasia $(5 \%)$, nodular conjunctivitis (4\%), dermoid (4\%), and primary acquired melanosis (3\%). ${ }^{4}$ The malignant conjunctival tumors consisted of melanomas $(2.2 \%)$ and lymphomas $(1.1 \%){ }^{4}$ A malignant melanoma was found in 18 of 806 cases and were reported to be more common in the 15- to

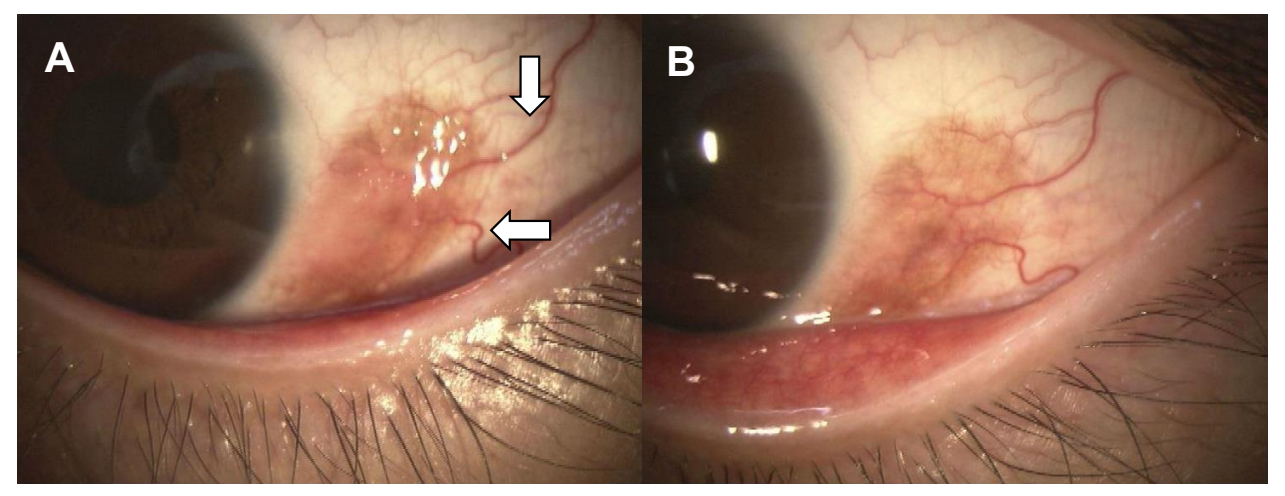

Figure 2 Slit-lamp biomicroscopic findings in Case 2. (A) Conjunctival tumor with high degree of pigmentation can be seen at the corneal limbus. There is also mild edema and feeder vessels (arrows). (B) The edema is reduced after one month of treatment with epinastine eye drops. 


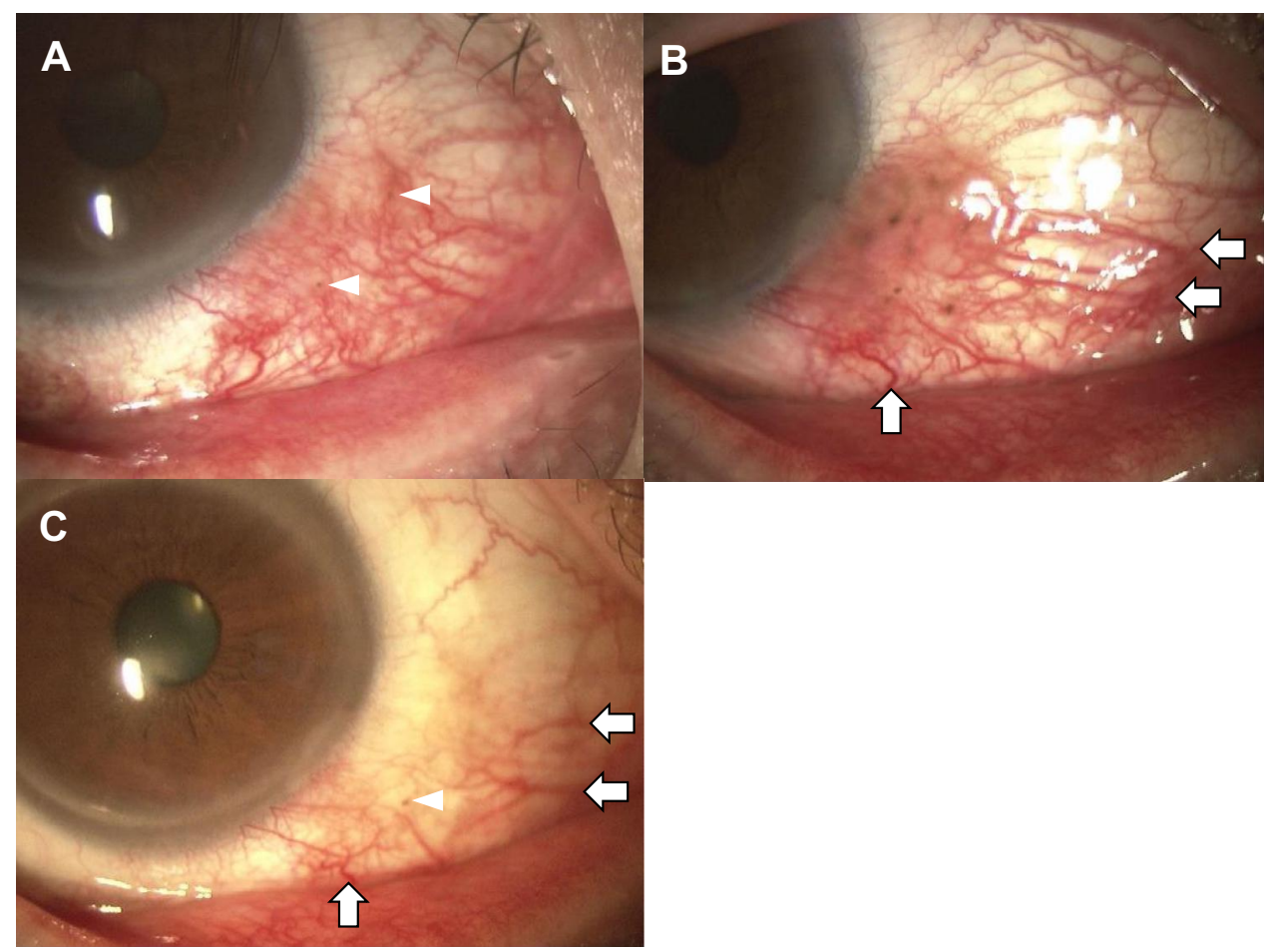

Figure 3 Slit-lamp biomicroscopic findings in Case 3. (A) There is marked hyperemia around the corneal limbus. Petechial pigmented lesion can be seen in the same area (arrow heads). (B) Photograph one month after treatment with topical epinastine and $0.1 \%$ fluorometholone eye drops. We can see an edematous neoplastic lesion with pigmentation, and it was accompanied by dilated feeder vessels (arrows). (C) One month after the treatment with topical epinastine and tacrolimus. The hyperemia, edema, and pigmentation are markedly improved and feeder vessels regressed (arrows).

21-year age group. When a patient presents with a conjunctival pigmented tumor, we have to determine whether it is a benign pigmentary lesion or a malignant melanoma. For an accurate diagnosis of a pigmented tumor, resection and histopathological examination is necessary. However, we believe that an excisional biopsy is not always necessary because it is possible to differentiate a malignant tumor from benign tumors based on the age of onset and its morphology such as a larger size, presence of hemorrhage, and the lack of intrinsic cysts. ${ }^{4}$ The age of our three patients were 8-, 14- and 34-year, and they all had a relatively small tumor without hemorrhages. Most importantly, the reduction in the tumor size with local treatment such as antiallergic and immunosuppressive agents suggested that the tumors were allergic in nature and not malignant.

Then, why did antiallergic drugs reduce the size of tumor in our three cases that suspected to be IJCN? Zamir et al reported on the histological characteristics of IJCN based on 47 biopsies of IJCN from 47 cases all $<20$-years-of-age. ${ }^{2}$ They reported that junctional nests and lymphocyte infiltrations were present in all cases. In addition, eosinophilic and plasma cell infiltration were found in $77 \%$ and $68 \%$ of cases, respectively. ${ }^{2}$ Levi-
Schaffer et al reported that there were many eosinophils and mast cells in the specimens of resected IJCN. ${ }^{5}$ These histopathological findings implied the existence of allergic reactions and chronic inflammations which are generally not seen in malignant tumors such as melanomas. ${ }^{3}$ The infiltration of lymphocytes, eosinophils, and mast cells associated with allergic reactions is known to be suppressed by antiallergic agents. Epinastine is an antihistamine that has anti-inflammatory properties that include $\mathrm{H}_{2}$-receptor antagonism, mast cell stabilization, and inhibition of cytokine production. It is used to treat allergic conjunctivitis of varying degrees of severity from mild to severe. The local administration of tacrolimus, which suppresses $\mathrm{T}$ cells and decreases the production of inflammatory mediators, has been reported to be an effective treatment of vernal conjunctivitis. We suggest that the infiltration of inflammatory cells was reduced by the topical antiallergic agent which may have contributed to the marked reduction in tumor size in our three IJCN cases.

Finally, the question arises on the mechanism causing the changes in the degree of pigmentation of the IJCN. It is well known that fibroblasts produce a stem cell factor (SCF) which activates epidermal melanocytes to proliferate which results in increased pigmentation under inflammatory conditions. ${ }^{6}$ 
Conjunctival melanocytes have some functional similarity to epidermal melanocytes. ${ }^{7}$ IJCN is a tumor with poor pigmentation, but SCF produced by fibroblast under allergic inflammatory conditions could be responsible for the increased pigmentation in IJCN. We believed that this was the most likely reason for the changes in the conjunctival pigmentation depending on the degree of allergic inflammation in our cases, especially in case 3 .

There is one limitation in this study. It has been reported that patients with vernal conjunctivitis have brown spotty pigmentations in the perilimbal bulbar conjunctiva. $^{8}$ Thus, excisional biopsies of the lesion are considered necessary to exclude the possibility of pigmentation associated with vernal conjunctivitis, but no excisional biopsies were performed in our cases. However, based on the age of onset, allergic predisposition, location of the conjunctival lesion and tumors were accompanied by feeder vessels, we considered that our cases were IJCN.

In conclusion, we reported three cases of a conjunctival tumor that we concluded to be IJCN, and they responded favorably to topical administration of antiallergic agents. Although it has been believed that tumor resection is the only treatment, local administration of antiallergic agents was found to reduce the size of the tumor. Considering that majority of patients with IJCN are younger than 20-years-of-age on whom tumor resection under local anesthesia is difficult, and the reports that the recurrence rate after simple excision of IJCN was $12 \%{ }^{9}$ treatment options other than resection should be considered. We believe that the local administration of an antiallergic agent has the advantage of allowing the patients to be followed until they can undergo tumor resection under local anesthesia while alleviating the cosmetic issues and should be presented to the child and family as an option before performing a tumor resection under general anesthesia.

\section{Ethics Approval and Informed Consents}

Ethical approval by the Ethics Committee of Mie University Hospital was waived due to the retrospective nature of the study considering that no personal identifying information of the patients was available from the records and the study was limited to the collection of clinical data.

\section{Patients Consent}

All patients or legal guardians provided informed consent for the case details, and also for any accompanying images to be published.

\section{Acknowledgments}

We thank Professor Emeritus Duco I. Hamasaki of the Bascom Palmer Eye Institute of the University of Miami (Miami, FL, USA) for critical discussion and final manuscript revisions.

\section{Author Contributions}

All authors made a significant contribution to the work reported, whether that is in the conception, study design, execution, acquisition of data, analysis and interpretation, or in all these areas; took part in drafting, revising or critically reviewing the article; gave final approval of the version to be published; have agreed on the journal to which the article has been submitted; and agree to be accountable for all aspects of the work.

\section{Funding}

Grant-in-Aid for Scientific Research C (MK, 18H02954 and 17K19721) from Ministry of Education, Culture, Sports, Science and Technology.

\section{Disclosure}

The authors report no conflicts of interest in this work.

\section{References}

1. Folberg R, Jakobiec FA, Bernardino VB, Iwamoto T. Benign conjunctival melanocytic lesions: clinicopathologic features. Ophthalmology. 1989;96(4):436-461.

2. Zamir E, Mechoulam H, Micera A, Levi-Schaffer F, Pe'er J. Inflamed juvenile conjunctival naevus: clinicopathological characterisation. $\mathrm{Br}$ J Ophthalmol. 2002;86(1):28-30. doi:10.1136/bjo.86.1.28

3. Choi EK, Chévez-Barrios P. Inflamed conjunctival nevi: histopathological criteria. Arch Pathol Lab Med. 2014;138(9):1242-1246. doi:10.5858/arpa.2013-0245-RS

4. Shields CL, Sioufi K, Alset AE, et al. Clinical features differentiating benign from malignant conjunctival tumors in children. JAMA Ophthalmol. 2017;135(3):215-224. doi:10.1001/jamaophtha lmol.2016.5544

5. Levi-Schaffer F, Micera A, Zamir E, et al. Nerve growth factor and eosinophils in inflamed juvenile conjunctival nevus. Invest Ophthalmol Vis Sci. 2002;43(6):1850-1856.

6. Fu C, Chen J, Lu J, et al. Roles of inflammation factors in melanogenesis (Review). Mol Med Rep. 2020;21(3):1421-1430.

7. Hu DN, McCormick SA, Seedor JA, Ritterband DC, Shah MK. Isolation, purification and cultivation of conjunctival melanocytes. Exp Eye Res. 2007;84(4):655-662. doi:10.1016/j.exer.2006.12. 003

8. Luk FO, Wong VW, Rao SK, Lam DS. Perilimbal conjunctival pigmentation in Chinese patients with vernal keratoconjunctivitis. Eye (London, England). 2008;22(8):1011-1014. doi:10.1038/sj. eye. 6702816

9. Thiagalingam S, Johnson MM, Colby KA, Zembowicz A. Juvenile conjunctival nevus: clinicopathologic analysis of 33 cases. $\mathrm{Am}$ J Surg Pathol. 2008;32(3):399-406. doi:10.1097/ PAS.0b013e31815143f3 


\section{Publish your work in this journal}

Clinical Ophthalmology is an international, peer-reviewed journal covering all subspecialties within ophthalmology. Key topics include: Optometry; Visual science; Pharmacology and drug therapy in eye diseases; Basic Sciences; Primary and Secondary eye care; Patient Safety and Quality of Care Improvements. This journal is indexed on PubMed

Submit your manuscript here: https://www.dovepress.com/clinical-ophthalmology-journal
Central and CAS, and is the official journal of The Society of Clinical Ophthalmology (SCO). The manuscript management system is completely online and includes a very quick and fair peer-review system, which is all easy to use. Visit http://www.dovepress.com/ testimonials.php to read real quotes from published authors. 